\title{
Etiology of childhood bilateral sensorineural hearing loss in
}

\section{Shandong province, China}

\section{Fan Jiang ${ }^{1 *}$, Hannah Kupper ${ }^{2}$, Tess Bright ${ }^{2}$, Wen-Zhe Qin ${ }^{1}$}

(1)

'School of Public Health, Shandong University, Jinan, China; Jiangfanqz@163.com (F.J.); qinwenzhe09@163.com (W.Q.)

${ }^{2}$ International Centre for Evidence in Disability, London School of Hygiene \& Tropical Medicine, London, UK; Hannah.kuper@lshtm.ac.uk (H.K.); Tess.bright@lshtm.ac.uk (T.B.)

*Correspondence: Jiangfanqz@163.com Tel.: +86-18053108811

School of Public Health, Shandong University, 44 Wen-hua- Road, Jinan, Shandong, 250012, China

\section{Conflicts of Interest}

The authors declare that there is no conflict of interests.

\section{Funding Statement}

We didn't receive funding for this study. 


\section{Abstract}

\section{Objectives}

The purpose of this study is to ascertain the etiology of bilateral sensorineural hearing loss (SNHL) in children aged $\leq 18$ years living in Shandong province.

\section{Methods}

Data were taken from a cross-sectional study which was conducted between 2015 and 2017.

The study included children aged $\leq 18$ years, recruited from special schools for children with hearing loss and from hearing rehabilitation centers in Shandong province of China. Children were screened for bilateral SNHL through audiological testing. Clinical examination, genetic testing and structured interviews were conducted for those children who were identified as having hearing loss to identify the potential cause.

\section{Results}

The etiology of bilateral SNHL in our sample was genetic in 874 (39.3\%), acquired in 650 (29.3\%) and unknown in 697 (31.4\%) children. Amongst children with acquired SNHL, the cause was maternal viral infection in $75(11.5 \%)$, perinatal factors in $238(36.6 \%)$, meningitis, measles and mumps in $146(22.5 \%)$, and ototoxic exposure in $117(18 \%)$. Among the children with genetic SNHL, only 44 (4.9\%) were identified as having syndromic hearing loss, and the remainder (95.1\%) were classified as non-syndromic hearing loss.

\section{Conclusion}

The findings indicated that nearly $30 \%$ of bilateral SNHL in Shandong province could be preventable through immunization, early prenatal diagnosis, proper treatment of infections and avoidance of prescription of ototoxic drugs. This finding emphasizes the need for 
programs aimed at improving the health services at primary and secondary levels of health care which will in turn prevent childhood hearing loss.

Keywords: SNHL; Etiology; Childhood; China.

\section{Introduction}

Hearing loss has become an important public health issue worldwide (Olusanya, Neumann, \& Saunders, 2014). According to the World Health Organization (WHO) estimate, across the world there are 16 million (12-26 million) children who have a hearing loss ( $>35 \mathrm{~dB} \mathrm{HL})$, and the global prevalence of hearing loss ( $>35 \mathrm{~dB} \mathrm{HL})$ among children 5-14 years of age is estimated to be $1.4 \%$ (Stevens et al., 2011). China is one of the most populous countries in the world, and among approximately 20 million babies born each year, around 60,000 are expected to have congenital hearing loss (WHO, 2010). A government survey reported that there were more than 1.7 million deaf and hard of hearing children (birth to age 18 years) in China (Li \& Prevatt, 2010).

Sensorineural hearing loss (SNHL) resulting from damage to the hair cells in the inner ear is the most common sensory deficit in humans (Prosser, Cohen, \& Greinwald, 2015). Bilateral SNHL has profound medical, social, and cultural ramifications (Smith, Bale Jr, \& White, 2005). Most notably, SNHL negatively impacts on the development of speech, language and cognitive skills in children, especially if it commences pre-lingually (Figueras, Edwards, \& Langdon, 2008; Walch, Anderhuber, Köle, \& Berghold, 2000). Untreated bilateral SNHL is also associated with slow progress in school, and difficulties in obtaining and performing 
effectively in jobs later in life (Fellinger, Holzinger, \& Pollard, 2012; Theunissen et al., 2014). Communication difficulties can also have lasting emotional and psychological consequences that can lead to feelings of isolation, loneliness and depression (Mason \& Mason, 2007; Stevenson et al., 2010). For instance, Li et al. (2010) found that the children and adolescents with SNHL in China reported significantly higher levels of total fears and anxieties than their peers with normal hearing. The impact on the family is also profound. Parents of children with bilateral SNHL must deal with specific challenges, are often at greater risk of stress, incur higher out-of-pocket expenses and lose more work days than other parents (Barton, Stacey, Fortnum, \& Summerfield, 2006; Yun et al., 2017). In addition to the effect on children and families, SNHL can have great economic effects on countries (Smith et al., 2005). Data from the 2015 Global Burden of Disease (GBD) database shows the estimated cost of childhood (birth to age 14 years) hearing loss to the health-care systems in China was \$ 7.86 billion (WHO, 2017).

The etiology of bilateral SNHL is traditionally classified as genetic, acquired and unknown (summarized in Table 1). Genetic SNHL is further classified as syndromic or non-syndromic and each of these is sub-categorised, depending upon the inheritance pattern. Most of these genetic phenotypes are not associated with a named syndrome or other anomaly (nonsyndromic), with only $15 \%$ to $30 \%$ occurring as part of a recognized syndrome (Prosser et al., 2015; Tranebjærg, 2005). Acquired or later acquired SNHL may be caused by prenatal, perinatal or postnatal environmental factors, such as cytomegalovirus (CMV), environmental exposures, meningitis, ototoxic medications or prematurity (Dietz, Löppönen, Valtonen, Hyvärinen, \& Löppönen, 2009; Mafong, Shin, \& Lalwani, 2002; Ogawa et al., 2007; Revello 
et al., 2015). However, the etiology of SNHL n can often not be determined conclusively for many children (Morzaria, Westerberg, \& Kozak, 2004).

The early identification of the etiological causes of SNHL is vital for prognosis, management, genetic counseling, prevention and effective rehabilitation (Prosser et al., 2015). However, SNHL remains underdiagnosed in children and etiology is not clarified in most developing countries, so these crucial opportunities are missed (Morzaria et al., 2004). Lack of information on the etiology of SNHL is also apparent in China, which hampers ability to plan prevention, treatment and rehabilitation services. Liu et al. (1993) did a survey about prevalence and etiology of profound deafness in the general population of Sichuan Province, China. The results showed that among 236 cases with profound hearing loss the etiology was most commonly genetic (43\%), followed by acquired $(35.6 \%)$ and unknown $(20.3 \%)$ causes. This study didn't divide the subjects into different groups according to age, so we lack data about the etiology of childhood hearing loss. Fu et al. (2010) studied the etiology of hearing loss in primary and middle school students in Hubei Province. Among 813 cases, 232 (28.5\%) were diagnosed with congenital hearing loss by pedigree analysis and $276(33.95 \%)$ cases were to have reported aminoglycoside-antibiotic-induced hearing loss. However, this study didn't report information on other acquired causes. Up-to-data information on the etiology of childhood hearing loss will be helpful for influencing local health policy and making plans for prevention and treatment, and reducing the prevalence of hearing loss. To fill the gap in the literature, the aim of the present study was to investigate the etiology of bilateral SNHL in children born in Shandong province in order to be able to promote preventative measures of 


\section{Materials and Methods}

The sample was derived from a cross-sectional survey which was conducted to estimate the etiology and health service needs of children ( $\leq 18$ years) with hearing loss. This study was conducted across in 17 special education schools and 22 hearing rehabilitation centers during 2015-2017. The hearing rehabilitation centers and the special schools were located in 17 administrative regions of Shandong province.

Study setting

Shandong province includes 140 counties belonging to 17 administrative regions. It has a population of 100 million, making it the second most populous province in China. According to the 2006 National Survey of Disability, 1.5 million people were estimated to have disabling hearing loss in Shandong province, including 15 thousand children under the age of 6 (National Bureau of Statistics, 2007). In 2017, the government health service reported that 2.3 thousand neonates and infants were identified with congenital or early childhood onset SNHL in Shandong province (Shandong Province Government Office, 2018). mainstream schools. The special schools are located in every administrative region of Shandong and are funded by local government. These schools provide education for children with hearing loss who were unable to benefit from hearing aids and have not receive cochlear implantation (CI). From September 2018, children under 6 years with profound hearing loss 
device) through basic medical insurance schemes (Shandong Province Government Office, 2018). However, before the improvements in medical insurance policies, access to CI was very limited for many children with hearing impairment, especially those living in rural and remote areas. As a consequence, many children with hearing loss were enrolled in special schools. Children with hearing impairment who receive hearing-aids or CI, can attend mainstream schools after passing an evaluation. A child with hearing impairment in mainstream schools may receive additional support from teachers. Children with CI receive training in rehabilitation centers after surgery. Usually, speech therapy begins with an emphasis on auditory training (detection, recognition, discrimination, and perception), followed by speech orthodontic treatment, articulation training, and language training according to the child's performance (Zhou, Chen, Shi, Wu, \& Yin, 2013). After two- or three-years' speech therapy, these children can transition to mainstream schools.

\subsection{Study participants}

All children aged $\leq 18$ years old registered either at the 17 special education schools or 22 hearing rehabilitation centers were considered for inclusion in the study. Children who met the following criteria were included: (1) aged $\leq 18$ years at time of interview; (2) diagnosed with bilateral SNHL. Children were excluded based on the following criteria: (1) residual hearing with Pure Tone Audiometry (PTA) $\leq 40 \mathrm{~dB}$ HL at two or more frequencies (500, 1000, 2000 and $4000 \mathrm{~Hz}$ ) or Auditory Steady State Response (ASSR) $\leq 40 \mathrm{~dB}$ HL two or more frequencies $(500,1000,2000,4000 \mathrm{~Hz})$; (2) had conductive, mixed or unilateral hearing loss. A total of 2221 children were included in the final analysis after excluding those had 
conductive, mixed or unilateral hearing loss $(n=115)$, age greater than stated criteria $(n=38)$, or children who did not complete the interview or physical tests $(n=20)$. The eligible 2221 children with bilateral SNHL were evaluated by the team of consultants, including four otolaryngologists, two audiologists, an audiology technician, an ophthalmologist, a genetic counselor, three nurses and three investigators.

We received ethical approval from Shandong Provincial ENT Hospital. Informed consent was obtained from the parents and children by the investigative team after explanation was given of the survey content and purpose.

\subsection{Audiological testing}

Auditory tests were performed by the audiologists and audiometric data for each child was recorded by the investigators. The severity of loss was determined using WHO (2016) classification in the better hearing ear with mild hearing loss at 26 to $40 \mathrm{~dB} \mathrm{HL}$, moderate loss at 41 to $60 \mathrm{~dB} \mathrm{HL}$, severe loss at 61 to $80 \mathrm{~dB} \mathrm{HL}$, and profound loss at greater than $81 \mathrm{~dB} \mathrm{HL}$. Depending upon the children's age and cooperation, PTA, ASSR and Pediatric Behavioral Audiometry (PBA) (including Behavioral Observation Audiometry, Visual Reinforcement Audiometry and Play Audiometry) were performed. For children 6 years old and above, PTA (at 500, 1000, 2000, and $4000 \mathrm{~Hz}$ ) data were collected. The tests were conducted in the quietest rooms available in local special schools and rehabilitation centers. For children aged less than 6 years old, PBA and ASSR were done in all cases. These procedures were performed at local hospitals. For children with cochlear implants, the hearing level before surgery was obtained from medical records of children, with the authorization of parents. 
Information about the children was collected using a standardized questionnaire form, which was completed by parents or teachers with support from the interviewers. Questionnaires covered medical history, family history and other details relevant to the hearing loss (e.g. prenatal, perinatal and postnatal history). Parents were asked about a family history of hearing loss, and, if possible, a pedigree was created. This information was gathered by the otolaryngology team, recorded by the specialized investigators and analyzed by the genetics team.

\subsection{Clinical examination}

Clinical examinations were performed by the otolaryngology team and ophthalmologist.

There are more than 200 different syndromes known to include hearing loss, and up to $30 \%$ of genetic hearing loss in children can be attributed to syndromic forms (Petit, 1996). Therefore, special attention was given to congenital findings that were known to be associated with syndromic hearing loss. The clinical examination included a detailed examination of the ears with an otoscope and, if necessary, an operating microscope. Specific etiologic findings evaluated were the shape and position of the pinna, patency of the external auditory canal (EAC), presence of otitis media, and abnormalities of the tympanic membrane. An ocular examination and craniofacial examination were also performed. Finally, other congenital anomalies such as pigmentation abnormalities of skins or abnormalities of limbs or stature were noted. 
All children with hearing loss were offered a genetic test for GBJ2, SLC26A4 and mitochondrial DNA12SrRNAA1555G. The literature on mutation hot spots of Chinese people with non-syndromic hearing loss indicates that mutations in GJB2 gene, SLC26A4 gene and $1555 \mathrm{~A}>\mathrm{G}$ mutation in mitochondrial DNA are likely to be common, and these were consequently the focus of our tests (Yuan et al., 2009). A 3-5 mL blood sample was taken from the antecubital vein in a vial containing Ethylenediaminetetraacetic acid (EDTA). The vial of blood was labeled with a secure identification number and refrigerated on site, pending transport back to the Shandong Provincial ENT hospital. On arrival in the laboratory, the blood sample was centrifuged to remove the leukocyte layer for DNA extraction.

\subsection{Diagnosis classification}

The audiometric data, clinical findings, genetic analysis results and risk factors were then reviewed by the team of consultants including an otolaryngologist, an audiologist, an ophthalmologist and a genetic counselor to determine the etiology of bilateral SNHL. Many are unclear and more than one cause may exist (Billings \& Kenna, 1999). In our study, an identified cause was given priority over the presence of risk factors. When dual or multiple causes existed, the evaluations of main cause were performed by the whole team, and based on the physicians' best judgement. 
213 Children with genetic causes were stratified into non-syndromic and syndromic groups.

214 Inclusion in a non-syndromic subgroup was determined by two factors: an identified mutation

215 in the hearing loss sensitive genes, and/or one or more close relatives with hearing loss.

216 Acquired SNHL was stratified into prenatal, perinatal and postnatal. Prenatal etiologies

217 include rubella, CMV, toxoplasma, herpes, syphilis, pregnancy-induced hypertension and

218 pesticide exposure. Perinatal etiologies include neonatal complications (hyperbilirubinemia,

219 asphyxia, prematurity, low birth weight), hydrocephalus and neonatal pneumonia. Postnatal

220 etiologies include meningitis, measles, mumps, trauma, otitis media, ototoxic drugs. There is

221 an overlap between the groups of ototoxicity and genetic mutation. Genetic mutation of DNA

222 12SrRNA A1555G is related to aminoglycoside antibiotics-induced deafness, which can

223 cause genetic susceptibility to aminoglycoside ototoxicity. The team of consultants classified

224 the children who had these two risk factors into the genetic etiology group.

225 The etiology of hearing loss was defined as unknown if there were no evidence for specific

226 risk factors, gene mutation or systemic syndromes.

227 3. Results

A total of 2221 children with bilateral SNHL were included in this study. The age of children ranged from 1 to 18 years old, $42.6 \%$ were girls and $63.6 \%$ lived in rural areas. The degree of 
in Table 2. In $874(39.3 \%)$ of the children, the cause was genetic factors. Acquired hearing loss was responsible for 650 cases (29.3\%) and unknown factors for 697 (31.4\%).

\subsection{Genetic etiology}

Genetic etiology was stratified into non-syndromic and syndromic subgroups (Table 3). 830 (95.1\%) children were classified as non-syndromic, with 631 (72.2\%) children found to have a genetic mutation, $44(5.1 \%)$ children had a family history, and $155(17.8 \%)$ children had both a genetic mutation and family history. In the 786 children who had a genetic mutation, we found $412(52 \%)$ had mutation in the GJB2 and $310(39 \%)$ had mutation for the

SLC26A4. A mutation of A1555G was seen in $64(8 \%)$ children. The syndromic SNHL group consisted of $44(5 \%)$ cases, including 35 children with (4\%) Waardenburg syndrome, two (0.2\%) Down syndrome, three (0.3\%) Goldenhar syndrome, two (0.2\%) Brueghel syndrome and two $(0.2 \%)$ Mobius syndrome.

\subsection{Systemic abnormalities}

Including the children with syndromic SNHL, systemic abnormalities were seen in 222 cases (Table 4). 14 children were noted to have skeletal development restriction, two children had spinal diseases, 18 children had intellectual impairment and 36 children had reported leukodystrophy. Significant ocular abnormalities were found in 55 children, including amblyopia, strabismus, ocular dysplasia, juvenile cataracts and 25 children had heterochromia iridis. Hypertelorism were seen in four children and five had high myopia. Congenital heart disease was observed in 16 children, with three children presenting with pulmonary stenosis. 
Two children had kidney malformation. Facial dysmorphism reported in 12 children with six children presenting with a history of cleft palate and six had cleft lip. Freckles could be found in 19 children and five children had distinct grey hair. These abnormalities can help us to identify the syndromic SNHL at an early period.

\subsection{Acquired etiology}

Acquired SNHL was detected in 650 children (Table 5). Prenatal risk factors were observed in $113(17.4 \%)$ children. The most common infection type was maternal infection during pregnancy. A total of $36(5 \%)$ mothers had rubella infection during pregnancy, six $(0.8 \%)$ reported a cytomegalovirus (CMV) infection, two (0.3\%) had toxoplasma infection, 24 (3.7\%) reported herpes virus infection and seven (1.1\%) reported syphilis infection. In addition, three $(0.5 \%)$ mothers reported pesticide exposure and 35 (5.4\%) reported pregnancy-induced hypertension.

Perinatal causes accounted for $36.8 \%$ of bilateral SNHL in these children. Among these, 68 $(11 \%)$ children reported neonatal complication (hyperbilirubinemia, asphyxia, prematurity, low birth weight), nine (1.4\%) had hydrocephalus and 20 (3.2\%) children had neonatal pneumonia. Exposure to ototoxic drugs was the largest cause in this group and occurred in $117(19 \%)$ cases in the postnatal subgroup. Ototoxic drugs were used to treat infections in a large percentage of children, with a known history of gentamicin exposure in 92 (15\%) children, kanamycin exposure in 13 (2\%) children and streptomycin exposure in $12(2 \%)$ children. 41 children who were found to have mutation at mitochondrial DNA 12SrRNA 
genetic etiology (above). A history of meningitis was noted in $86(13.2 \%)$ children, mumps and measles were recorded in $60(9.2 \%)$ children. Finally, $36(5 \%)$ children had a history of head trauma before the onset of bilateral SNHL.

\section{Discussion}

The cause of bilateral SNHL in children is often not determined in developing countries (Morzaria et al., 2004; Sun, Wei, Yu, Wang, \& Liang, 2008). The main cause of the data gap is that the diagnostic search for an underlying cause can be expensive, time-consuming, and inconclusive, and that appropriately trained clinicians needed to make the diagnosis may not be available (Mulwafu, Kuper, \& Ensink, 2016; Stevens et al., 2011). However, up-to-date information on the etiology of bilateral SNHL is needed to direct strategies for avoiding and treating those preventable causes (Feder et al., 2017). Our study in Shandong, China, found that the biggest cause of bilateral SNHL in 2221 children was genetic (39.4\%), while fewer cases were of acquired (29.3\%) or unknown etiology (31.3\%). Genetic causes included $95.1 \%$ non-syndromic etiology and $4.9 \%$ with syndromic etiology. Of the acquired causes of hearing loss, we found $17.4 \%$ prenatal, $36.6 \%$ perinatal and $46 \%$ postnatal acquired etiology.

WHO (2016) estimated that $40 \%$ of childhood hearing loss was caused by genetic factors. In previous studies from developing countries, the etiology of childhood hearing loss was estimated as genetic in 13\%-63\%, and the cause remained unknown in $18-53 \%$ and was nongenetic in the remainder (Dereköy, 2000; Egeli et al., 2003; Khabori, 2004; Silan et al., 2004; Zakzouk \& Al-Anazy, 2002). We found that $39.3 \%$ of cases were genetic, which was consistent with previous literature on mutation hot spots in the Chinese population with non- 
syndromic hearing loss (Yuan et al., 2009). Of the 875 children with a genetic SNHL, just $4.9 \%$ were syndromic. This figure contrasts with the prevailing views on genetic SNHL distribution, as researchers suggest that up to $30 \%$ of all genetic hearing loss is syndromic (Smith et al., 2005). One explanation for this discrepancy is the structure of the special educational schools and hearing rehabilitation centers in our setting, which do not provide suitable facilities for the children who have multiple disabilities (i.e. syndromic cases) and so they may have been excluded from our sample.

WHO (2016) estimates that about $60 \%$ of hearing loss is due to preventable causes and this proportion is higher in developing countries $(75 \%)$. Among the causes of preventable hearing loss, neonatal complications account for $17 \%$ of childhood hearing loss (WHO, 2016).

Neonatal complications were estimated to be the cause of bilateral SNHL in $9 \%$ of our study sample, compared to a slightly higher estimate of $12.1 \%-17.3 \%$ in previous studies of developing countries (Egeli et al., 2003; Khabori, 2004; Zakzouk \& Al-Anazy, 2002). Another difference from previous reports was the lower prevalence of TORCH infectious (toxoplasmosis, other, rubella, cytomegalovirus and herpes) in those with bilateral SNHL from $7.6 \%$ to $23.8 \%$, compared to our figure of $4.2 \%$ (Dereköy, 2000; Zakzouk \& Al-Anazy, 2002). The lower proportion of hearing loss due to perinatal and prenatal factors in our study may be attributed to improvements in pre and perinatal care and the emphasis on timely TORCH examination and vaccination for pregnant women before their pregnancy in In previous studies, infectious diseases (meningitis, measles and mumps) were found to be 
one of the most common causes of bilateral SNHL in children (Smith et al., 2005). In particular, previous studies have reported that meningitis accounts for $21 \%-43 \%$ of acquired SNHL in developing countries (Dereköy, 2000; Egeli et al., 2003; Khabori, 2004; Sajjad, Khattak, Bunn, \& Mackenzie, 2008; Silan et al., 2004; Zakzouk \& Al-Anazy, 2002). Measles is a less common cause ranging from $11 \%$ to $29 \%$ of acquired SNHL (Dereköy, 2000; Egeli et al., 2003; Sajjad et al., 2008; Silan et al., 2004; Zakzouk \& Al-Anazy, 2002). In our study, infectious disease accounted for $21 \%$ of acquired factors and $2.7 \%$ of the total causes, making our findings consistent with earlier studies. A clear implication is that infectious diseases should be avoided to reduce SNHL. Indeed, WHO (2016) suggests that over 19\% of childhood hearing loss could be avoided through immunization against rubella and meningitis. Strengthening immunization programmes will therefore be effective at prevention of viral infection of children that lead to hearing loss, such as congenital rubella, meningitis, mumps and measles (Swamy \& Heine, 2015). However, according to the vaccination report, the estimated vaccination rate of MMR (Measles, Mumps and Rubella Combined Attenuated Live Vaccine) in rural area of China was just 50\%-60\%, with a much lower level expected for those in remote areas (Li et al., 2017). These low coverage figures may explain the high frequency of measles and mumps in our study.

It is noteworthy, that in our study the extent of ototoxic exposure in children with SNHL (5.3\%) was lower than previously reported (Fu et al., 2010). The unregulated use of ototoxic drugs has been a major problem in China (Yun et al., 2017). In particular, the aminoglycoside gentamicin has been widely used in China because of its low cost (Jian, Deng, \& Sun, 2015). Community based use of ototoxic medicine is difficult to track; however, studies have shown 
that $30 \%$ to $40 \%$ of inpatient use of ototoxic drug in Chinese children may be inappropriate (Kumana, Li, Kou, \& Chan, 1989). In the past 15 years, China implemented legislation to restrict the sale and use of ototoxic medicines (Gong et al., 2018). However, the higher frequency of ototoxic exposure reported in rural compared to urban areas in our study highlights the need to strengthen publicity and education about the harmfulness of ototoxicity drugs in rural area of China.

In general, our data indicates that nearly $30 \%$ childhood SHNL in Shandong province could be prevented. This study highlights the importance of improving maternal and neonatal care, including strengthening the national immunization programme to ensure widespread coverage, avoiding ototoxic drugs, and early diagnosis and proper treatment of prenatal and postnatal infection in order to reduce the incidence of SNHL in children. Targeted genetic tests may also be helpful for families to understand what is happening and to provide genetic counselling which may help to decreasing the prevalence of genetic SNHL (Wormald, Viani, Lynch, \& Green, 2010). Genetic screening for a specific mitochondrial mutation during pregnancy could offer a strategy of minimizing bilateral SNHL in babies from exposure to avoidable risk factors such as neonatal use of aminoglycoside antibiotics. For the children who have large vestibular aqueduct syndrome (LAVS), the genetic screening may enable interventions to protect against trauma which could lead to SNHL (Xiang et al., 2017). There may also be an important role for genetic testing in all newborns that do not pass newborn hearing screening and their lineal relatives, especially the people who have family history of hearing loss. In doing so, the information could assist in establishing the prevalence and links between gene mutation and hearing loss in China. 
This study has some limitations that need to be taken in to account when interpreting results.

Firstly, the children screened in the study may be influenced by selection bias in that some children with bilateral SNHL may not attend specialist schools or rehabilitation centers, such as children with multiple disabilities. Another consideration is that WHO (2016) classifies disabling hearing loss as a hearing loss greater than $30 \mathrm{~dB}$ HL in the better hearing ear in children, whereas in our study we only included children with hearing loss $\geq 40 \mathrm{~dB}$ HL to allow comparison with previous studies. As such, some forms of milder SNHL were not included in our study. Consequently, this group of children does not yield reliable information about the etiology of childhood SNHL in the entire population. Secondly, there is the potential for bias in collecting data by questionnaire. However, we tried our best in quality control to make sure consistency in assessment. Thirdly, we are aware that an exact classification of possible causes is problematic and that there are coexistent risk factors in this group of children. This may have resulted in the underestimation of some causes of bilateral SNHL among children. However, to the best of our knowledge, the data used in our study is the most recent and largest study on childhood SNHL in China.

\section{Conclusion}

In conclusion, the most common causes of bilateral SNHL in children aged $\leq 18$ years in Shandong province were genetic non-syndromic (37.3\%), unknown (31.4\%), postnatal $(13.5 \%)$, perinatal $(10.7 \%)$, prenatal $(5.1 \%)$, and genetic syndromic $(2 \%)$. That means that nearly $30 \%$ of cases of SNHL in childhood in this study could be preventable or treatable at primary and secondary levels of health care. Consequently, we have to improve physician 
awareness and develop guidelines for medical evaluation of pediatric SNHL. Governments, public health agencies, social service organizations, educational institutions and civil society groups all need to collaborate in this endeavor. Comprehensive services are needed to support children with SNHL, so that they are included in school and wider society, and are able to maximize their quality of life and opportunities in life.

\section{Declarations of interest}

The authors declare that there is no conflict of interests.

\section{Acknowledgment}

We would like to thank all study participants for their time to be interviewed. We also thank the support of Shandong ENT hospital and School of Public Health, Shandong University.

\section{Reference}

Barton, G. R., Stacey, P. C., Fortnum, H. M., \& Summerfield, A. Q. (2006). Hearingimpaired children in the United Kingdom, II: Cochlear implantation and the cost of compulsory education. Ear and hearing, 27(2), 187-207. doi:10.1097/01.aud.0000202348.77016.1f

Billings, K. R., \& Kenna, M. A. (1999). Causes of pediatric sensorineural hearing loss: yesterday and today. Archives of Otolaryngology-Head \& Neck Surgery, 125(5), 517521. doi:10.1001/archotol.125.5.517

Dereköy, F. S. (2000). Etiology of deafness in Afyon school for the deaf in Turkey. International Journal of Pediatric Otorhinolaryngology, 55(2), 125-131. doi:https://doi.org/10.1016/S0165-5876(00)00390-6

Dietz, A., Löppönen, T., Valtonen, H., Hyvärinen, A., \& Löppönen, H. (2009). Prevalence and etiology of congenital or early acquired hearing impairment in Eastern Finland. International Journal of Pediatric Otorhinolaryngology, 73(10), 1353-1357. doi:https://doi.org/10.1016/j.ijporl.2009.06.009

Egeli, E., Çiçekci, G., Silan, F., Öztürk, Ö., Harputluoğlu, U., Onur, A., Egeli, A., \& Yildiz, A. (2003). Etiology of deafness at the Yeditepe school for the deaf in Istanbul. 
International Journal of Pediatric Otorhinolaryngology, 67(5), 467-471. doi:https://doi.org/10.1016/S0165-5876(03)00002-8

Feder, K. P., Michaud, D., McNamee, J., Fitzpatrick, E., Ramage-Morin, P., \& Beauregard, Y. (2017). Prevalence of hearing loss among a representative sample of Canadian children and adolescents, 3 to 19 years of age. Ear and hearing, 38(1), 720. doi:10.1097/AUD.0000000000000345

Fellinger, J., Holzinger, D., \& Pollard, R. (2012). Mental health of deaf people. The Lancet, 379(9820), 1037-1044. doi:https://doi.org/10.1016/S0140-6736(11)61143-4

Figueras, B., Edwards, L., \& Langdon, D. (2008). Executive function and language in deaf children. Journal of Deaf Studies and Deaf Education, 13(3), 362-377. doi:https://doi.org/10.1093/deafed/enm067

Fu, S., Chen, G., Dong, J., \& Zhang, L. (2010). Prevalence and etiology of hearing loss in primary and middle school students in the Hubei Province of China. Audiology and Neurotology, 15(6), 394-398. doi:https://doi.org/10.1159/000307346

Gong, R., Hu, X., Gong, C., Long, M., Han, R., Zhou, L., Wang, F., \& Zheng, X. (2018). Hearing loss prevalence and risk factors among older adults in China. International journal of audiology, 57(5), 354-359. doi:https://doi.org/10.1080/14992027.2017.1423404

Jian, X., Deng, Z., \& Sun, Y. (2015). Aminoglycoside gentamicin research: fundamental progress and new application prospects. Sheng wu gong cheng xue bao=Chinese journal of biotechnology, 31(6), 829-844.

Khabori, M. A. (2004). Causes of severe to profound deafness in Omani paediatric population. International Journal of Pediatric Otorhinolaryngology, 68(10), 13071313. doi:https://doi.org/10.1016/j.ijporl.2004.05.002

Kumana, C., Li, K., Kou, M., \& Chan, S. (1989). Cephalosporin and aminoglycoside utilization in different parts of the world. Journal of Antimicrobial Chemotherapy, 24(6), 1001-1010. doi:https://doi.org/10.1093/jac/24.6.1001

Li, H., \& Prevatt, F. (2010). Deaf and hard of hearing children and adolescents in China: their fears and anxieties. American Annals of the deaf, 155(4), 458-466. doi:https://www.jstor.org/stable/26235084

Li, R., Cheng, S., Luo, C., Rutherford, S., Cao, J., Xu, Q., Liu, X., Liu, Y., Xue, F., \& Xu, Q. (2017). Epidemiological characteristics and spatial-temporal clusters of mumps in Shandong Province, China, 2005-2014. Scientific reports, 7, 46328. doi:https://xs.scihub.ltd/https://doi.org/10.1038/srep46328

Liu, X., Xu, L., Zhang, S., \& Xu, Y. (1993). Prevalence and aetiology of profound deafness in the general population of Sichuan, China. The Journal of Laryngology \& Otology, 107(11), 990-993. doi: https://doi.org/10.1017/S0022215100125071

Mafong, D. D., Shin, E. J., \& Lalwani, A. K. (2002). Use of laboratory evaluation and radiologic imaging in the diagnostic evaluation of children with sensorineural hearing loss. The Laryngoscope, 112(1), 1-7. doi:https://doi.org/10.1097/00005537200201000-00001

Mason, A., \& Mason, M. (2007). Psychologic impact of deafness on the child and adolescent. Primary Care: Clinics in Office Practice, 34(2), 407-426. doi:https://doi.org/10.1016/j.pop.2007.04.003 
Morzaria, S., Westerberg, B. D., \& Kozak, F. K. (2004). Systematic review of the etiology of bilateral sensorineural hearing loss in children. International Journal of Pediatric Otorhinolaryngology, 68(9), 1193-1198. doi:https://doi.org/10.1016/j.ijporl.2004.04.013

Mulwafu, W., Kuper, H., \& Ensink, R. (2016). Prevalence and causes of hearing impairment in Africa. Tropical medicine \& international health, 21(2), 158-165. doi:https://doi.org/10.1111/tmi.12640

National Bureau of Statistics. (2007). Main Data Bulletin of the Second National Sample Survey of Disabled Persons. Retrieved from http://www.stats.gov.cn/tjsj/ndsj/shehui/2006/html/fu3.htm

Ogawa, H., Suzutan, T., Baba, Y., Koyano, S., Nozawa, N., Ishibashi, K., Fujieda, K., Inoue, N., \& Omori, K. (2007). Etiology of severe sensorineural hearing loss in children: independent impact of congenital cytomegalovirus infection and GJB2 mutations. The Journal of infectious diseases, 195(6), 782-788. doi:https://doi.org/10.1086/511981

Olusanya, B. O., Neumann, K. J., \& Saunders, J. E. (2014). The global burden of disabling hearing impairment: a call to action. Bulletin of the World Health Organization, 92(5), 367-373. doi:https://doi.org/10.2471/BLT.13.128728

Petit, C., . (1996). Genes responsible for human hereditary deafness: symphony of a thousand. Nature Genetics, 14(4), 385-391. doi:https://xs.scihub.ltd/https://doi.org/10.1038/ng1296-385

Prosser, J. D., Cohen, A. P., \& Greinwald, J. H. (2015). Diagnostic evaluation of children with sensorineural hearing loss. Otolaryngologic Clinics of North America, 48(6), 975-982. doi:https://doi.org/10.1016/j.otc.2015.07.004

Revello, M. G., Tibaldi, C., Masuelli, G., Frisina, V., Sacchi, A., Furione, M., Arossa, A., Spinillo, A., Klersy, C., Ceccarelli, M., Gerna, G., \& Todros, T. (2015). Prevention of Primary Cytomegalovirus Infection in Pregnancy. Ebiomedicine, 2(9), 1205-1210. doi:https://doi.org/10.1016/j.ebiom.2015.08.003

Sajjad, M., Khattak, A., Bunn, J., \& Mackenzie, I. (2008). Causes of childhood deafness in Pukhtoonkhwa Province of Pakistan and the role of consanguinity. The Journal of Laryngology \& Otology, 122(10), 1057-1063. doi: https://doi.org/10.1017/S0022215108002235

Shandong Province Government Office. (2018). Notice of the Peoples' Government of Shandong Province on the Establishment of Rehabilitation Assistance System for Disabled Children. Retrieved from http://www.shandong.gov.cn/art/2018/9/30/art_2259_28657.html

Silan, F., Demirci, L., Egeli, A., Egeli, E., Onder, H. I., Ozturk, O., \& Unal, Z. S. (2004). Syndromic etiology in children at schools for the deaf in Turkey. International Journal of Pediatric Otorhinolaryngology, 68(11), 1399-1406. doi:https://doi.org/10.1016/j.ijporl.2004.05.007

Smith, R. J., Bale Jr, J. F., \& White, K. R. (2005). Sensorineural hearing loss in children. The Lancet, 365(9462), 879-890. doi:https://doi.org/10.1016/S0140-6736(05)71047-3

Stevens, G., Flaxman, S., Brunskill, E., Mascarenhas, M., Mathers, C. D., \& Finucane, M. (2011). Global and regional hearing impairment prevalence: an analysis of 42 
studies in 29 countries. The European Journal of Public Health, 23(1), 146-152. doi:https://doi.org/10.1093/eurpub/ckr176

Stevenson, J., McCann, D., Watkin, P., Worsfold, S., Kennedy, C., \& Team, H. O. S. (2010). The relationship between language development and behaviour problems in children with hearing loss. Journal of Child Psychology and Psychiatry, 51(1), 77-83. doi:https://doi.org/10.1111/j.1469-7610.2009.02124.x

Sun, X., Wei, Z., Yu, L., Wang, Q., \& Liang, W. (2008). Prevalence and etiology of people with hearing impairment in China. Zhonghua liu xing bing xue za zhi=Zhonghua liuxingbingxue zazhi, 29(7), 643-646.

Swamy, G. K., \& Heine, R. P. (2015). Vaccinations for pregnant women. Obstetrics and gynecology, 125(1), 212-226. doi:10.1097/AOG.0000000000000581

Theunissen, S. C., Rieffe, C., Netten, A. P., Briaire, J. J., Soede, W., Schoones, J. W., \& Frijns, J. H. (2014). Psychopathology and its risk and protective factors in hearingimpaired children and adolescents: A systematic review. JAMA pediatrics, 168(2), 170-177. doi:https://doi.org/10.1001/jamapediatrics.2013.3974

Tranebjærg, L. (2005). Hereditary hearing loss - the updated resource book more needed than ever. European Journal of Human Genetics, 13(7), 889-890. doi:https://xs.scihub.ltd/https://doi.org/10.1038/sj.ejhg.5201396

Walch, C., Anderhuber, W., Köle, W., \& Berghold, A. (2000). Bilateral sensorineural hearing disorders in children: etiology of deafness and evaluation of hearing tests. International Journal of Pediatric Otorhinolaryngology, 53(1), 31-38. doi:https://doi.org/10.1016/S0165-5876(00)00307-4

World Health Organization. (2010). Newborn and infant hearing screening: Current issues and guiding principles for action. Outcomes of a WHO informal consultation held at World Health Organization headquarters (9-10 November 2009). Geneva, Switzerland.

World Health Organization. (2016). Childhood hearing loss: strategies for prevention and care. Retrieved from https://apps.who.int/iris/bitstream/handle/10665/204632/9789241510325_eng.pdf

World Health Organization. (2017). Global costs of unaddressed hearing loss and costeffectiveness of interventions: $a$ WHO report, 2017: World Health Organization.

Wormald, R., Viani, L., Lynch, S. A., \& Green, A. J. (2010). Sensorineural hearing loss in children. Irish medical journal, 103(2), 51-54.

Xiang, Y., Li, H., Xu, X., Xu, C., Che, n. C., Lin, X., \& Tang, S. (2017). Mutation analysis and prenatal diagnosis for 12 families affected with hereditary hearing loss and enlarged vestibular aqueduct. Chinese Journal of Medical Genetics, 34(3), 336-341. doi:10.3760/cma.j.issn.1003-9406.2017.03.005

Yuan, Y., You, Y., Huang, D., Cui, J., Wang, Y., Wang, Q., Yu, F., Kang, D., Yuan, H., \& Han, D. (2009). Comprehensive molecular etiology analysis of nonsyndromic hearing impairment from typical areas in China. Journal of Translational Medicine, 7(1), 79. doi:https://doi.org/10.1186/1479-5876-7-79

Yun, C., Wang, Z., Gao, J., He, P., Guo, C., Chen, G., \& Zheng, X. (2017). Prevalence and social risk factors for hearing impairment in chinese children - a national survey. International journal of environmental research and public health, 14(1), 88. 

doi:https://doi.org/10.3390/ijerph14010088

Zakzouk, S. M., \& Al-Anazy, F. (2002). Sensorineural hearing impaired children with unknown causes: a comprehensive etiological study. International Journal of Pediatric Otorhinolaryngology, 64(1), 17-21. doi:https://doi.org/10.1016/S01655876(02)00029-0

Zhou, H., Chen, Z., Shi, H., Wu, Y., \& Yin, S. (2013). Categories of auditory performance and speech intelligibility ratings of early-implanted children without speech training. Plos One, 8(1), e53852. doi:https://doi.org/10.1371/journal.pone.0053852 
Table 1 The most common etiologies of sensorineural hearing loss

\begin{tabular}{ll}
\hline & Causes \\
\hline Prenatal & $\begin{array}{l}\text { Rubella, toxoplasmosis, cytomegalovirus (CMV), herpes, syphilis, } \\
\text { pregnancy-induced hypertension, pesticide exposure }\end{array}$ \\
Perinatal & $\begin{array}{l}\text { Hyperbilirubinemia, asphyxia,prematurity and low birth weight, } \\
\text { hydrocephalus ,neonatal pneumonia }\end{array}$ \\
Postnatal & meningitis, measles and mumps, trauma, ototoxicity, Otitis media \\
\hline
\end{tabular}

Table 2. Distribution of cases according to the etiologies of hearing impairment

\begin{tabular}{cccccc}
\hline Hearing loss criteria & & Genetic & Acquired & Unknown & Total \\
\hline Gender & Girl & 362 & 276 & 309 & 947 \\
& Boy & 512 & 374 & 388 & 1274 \\
Residence & Urban & 373 & 194 & 242 & 809 \\
& Rural & 501 & 456 & 455 & 1412 \\
\multirow{2}{*}{ Age } & $1 \sim$ & 239 & 169 & 101 & 509 \\
& $6 \sim$ & 295 & 255 & 847 \\
Degree of & Moderate & 20 & 186 & 341 & 865 \\
hearing & Severe & 117 & 54 & 51 & 125 \\
impairment & Profound & 737 & 66 & 48 & 231 \\
& & & 530 & 598 & 1865 \\
\hline
\end{tabular}


Table 3. Distribution of cases in genetic group

\begin{tabular}{llcc}
\hline & & Number of cases & Percentage \% \\
\hline \multirow{3}{*}{ Non-syndromic } & Identified genetic mutation & 631 & 72.2 \\
& Family history & 44 & 5.1 \\
& Both genetic mutation and & 155 & 17.8 \\
& family history & & \\
\hline \multirow{3}{*}{ Syndromic } & Waardenburg syndrome & 35 & 4 \\
& Down syndrome & 2 & 0.2 \\
& Goldenhar syndrome & 3 & 0.3 \\
& Brueghel syndrome & 2 & 0.2 \\
& Mobius syndrome & 2 & 0.2 \\
& & 874 & 100 \\
\hline
\end{tabular}

Table 4. Systemic abnormalities among cases

\begin{tabular}{ll}
\hline Name of systemic abnormalities & Number of cases \\
\hline Skeletal development restriction & 14 \\
Spinal diseases & 2 \\
Intellectual impairment & 18 \\
Leukodystrophy & 36 \\
Ocular abnormalities & 55 \\
Hypertelorism & 4 \\
High myopia & 5 \\
Congenital heart disease & 16 \\
Heterochromia iridis & 25 \\
Pulmonary stenosis & 3 \\
Kidney malformation & 2 \\
Facial dysmorphism & 12 \\
Freckles & 19 \\
Distinct grey hair & 5 \\
\hline
\end{tabular}


Table 5. Distribution of cases in acquired group

\begin{tabular}{|c|c|c|c|}
\hline & Name of disease & Number of cases & Percentage $(\%)$ \\
\hline \multirow{7}{*}{ Acquired/ prenatal } & Rubella & 36 & 5 \\
\hline & CMV & 6 & 0.8 \\
\hline & Toxoplasma & 2 & 0.3 \\
\hline & Herpes & 24 & 3.7 \\
\hline & Syphilis & 7 & 1.1 \\
\hline & Pregnancy-induced hypertension & 35 & 5.4 \\
\hline & Pesticide exposure & 3 & 0.5 \\
\hline \multirow{3}{*}{ Acquired/perinatal } & Neonatal complications & 209 & 32.2 \\
\hline & Hydrocephalus & 9 & 1.4 \\
\hline & Neonatal pneumonia & 20 & 3.2 \\
\hline \multirow{5}{*}{ Acquired/postnatal } & Meningitis & 86 & 13.2 \\
\hline & Measles and mumps & 60 & 9.2 \\
\hline & Trauma & 36 & 5 \\
\hline & Ototoxicity & 117 & 19 \\
\hline & & 650 & 100 \\
\hline
\end{tabular}

